\title{
CT chest with pulmonary angiography as a diagnostic tool in clinically suspected RT-PCR-negative COVID-19 pneumonia with pulmonary artery aneurysm
}

\author{
Aniket Agarwal', Niraj Kumar ${ }^{2}$ \\ From ${ }^{1}$ Senior Resident, Department of Radiology, ${ }^{2}$ Consultant, Department of Medicine, ABVIMS and Dr. RML Hospital, New Delhi, India
}

\begin{abstract}
COVID-19 caused by the SARS-CoV-2 strain of coronavirus was officially recognized as a pandemic by the World Health Organization on March 11, 2020. Most patients present with mild disease. However, elderly patients and those with coexisting comorbid conditions such as diabetes and hypertension are more likely to develop severe disease. Definitive diagnosis is by RT-PCR test and CT scan is an adjunct modality. By virtue of being a hypercoagulable state with cytokine storm and microthrombosis as key components in pathogenesis, additional finding of pulmonary thrombosis in such patients should increase diagnostic accuracy. We report an interesting case with clinical and radiological features supporting COVID-19 pneumonia such as patchy ground-glass opacities with consolidation in bilateral peripheral lung fields along with segmental pulmonary thrombosis. The conundrum in the case arises from the negative RT-PCR test and presence of pulmonary artery aneurysm which could be an incidental finding or sequelae of COVID-19, which remains to be studied.
\end{abstract}

Key words: COVID-19, CT pulmonary angiography, Cytokine storm, Microthrombosis

$\mathrm{C}$ oronavirus disease 2019 (COVID-19) is an infectious disease caused by severe acute respiratory syndrome coronavirus 2 (SARS-CoV-2), a strain of coronavirus. The outbreak was officially recognized as a pandemic by the World Health Organization (WHO) on March 11, 2020. The patients with COVID-19 present primarily with fever, myalgia or fatigue, and dry cough. Although most patients have a favorable prognosis, older patients and those with chronic underlying conditions such as diabetes and hypertension may have poor outcomes [1]. The patients with severe acute respiratory illness (SARI) may develop dyspnea and hypoxemia within 1 week after the onset of the disease, which may quickly progress to acute respiratory distress syndrome (ARDS) with or without other end-organ failures [2]. A definitive diagnosis of COVID-19 is by reverse transcriptase polymerase chain reaction (RT-PCR) test and computed tomography (CT) scan is an adjunct modality helpful in assessing complications.

We report a case of clinically suspected patient with COVID19 who developed ARDS and pulmonary thrombosis after hospital admission and was lost on the 14th day of hospital admission.

\section{Access this article online}

Received - 24 January 2021

Initial Review - 11 February 2021

Accepted - 01 March 2021

DOI: $10.32677 /$ IJCR.2021.v07.i03.002

\section{CASE REPORT}

A 53-year-old man, diagnosed case of hypertension and diabetes mellitus for 14 years, presented with history of undocumented fever, malaise, occasional dry cough, and breathlessness for 5 days in the month of May 2020 at the peak of the pandemic in our city. The general condition of the patient was poor.

At the time of admission, the blood pressure was 152/96, respiratory rate was $20 / \mathrm{min}$, and the temperature was $102.4^{\circ} \mathrm{C}$. The patient was hypoxic at admission $\left(\mathrm{PaO}_{2}\right.$ of 60.6 and $\mathrm{PaCO}_{2}$ of 52.2 with a pH of 7.145, and serum $\mathrm{HCO}_{3}$ of 18.2).

Blood sugars were uncontrolled at admission (HbAlc of 18). He also had a derangement of renal functions (creatinine of 2.9 with an estimated glomerular filtration rate of 19.6). Chest radiograph done on the day of admission revealed a right parahilar consolidation and a few patchy opacities in both lung fields (Fig. 1). Since the patient developed hemoptysis and continued to be hypoxic, a clinical diagnosis of pulmonary thromboembolism was considered. D-dimer was raised four times. Doppler of bilateral lower limbs revealed no evidence of deep venous thrombosis (DVT).

CT pulmonary angiography (CTPA) was done on day 5 for confirmation. CTPA revealed thrombosis in multiple segmental branches in both lung fields (right $>$ left) (Fig. 2a-h). A dumbbellshaped aneurysm measuring $2.8 \times 2 \mathrm{~cm}$ was also noted arising

Correspondence to: Dr. Aniket Agarwal, C-191, Anand Vihar, Delhi - 110092 , India. E-mail: aniketaggarwal@hotmail.com

(C) 2021 Creative Commons Attribution-NonCommercial 4.0 International License (CC BY-NC-ND 4.0). 

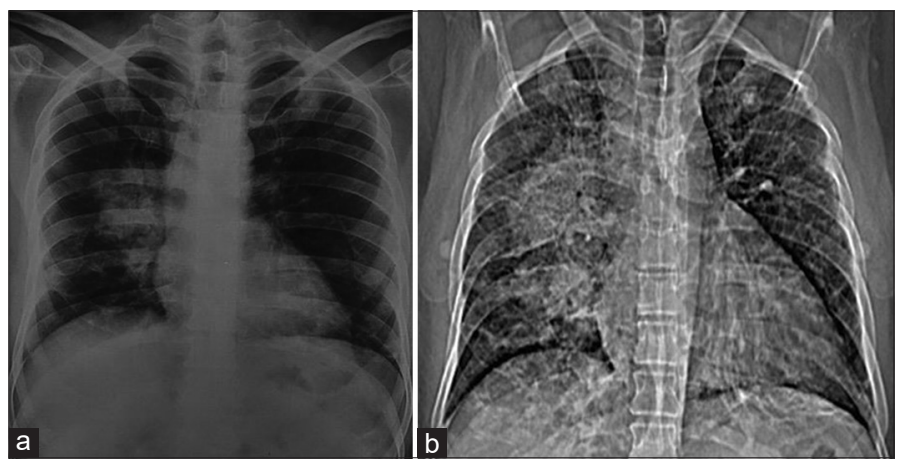

Figure 1: (a) Chest radiograph (at the time of admission) shows a large right parahilar consolidation with few ill-defined opacities in the left lower zone. (b) Topogram showing progression of right parahilar consolidation. Few other ill-defined areas of consolidation are also seen in the bolth lung fields.

from the superior segmental branch of the right interlobar pulmonary artery, just proximal to the level of the thrombosed segmental branch (Fig. 2c). There was a defect in the anterior wall of the aneurysm with surrounding consolidation (average Hounsfield Units 40) and ground-glass haze with intervening interlobular septal thickening suggestive of pulmonary hemorrhage (Fig. 3). There were no cavitation and no history of pulmonary tuberculosis. Areas of consolidation and groundglass opacities with interstitial thickening giving the appearance of crazy pavement were also seen in parahilar location in the right lung field and peripheral regions of both lung fields along with few centrilobular nodules (Fig. 3). There were mild bilateral pleural effusion and mild pericardial thickening. No significant lymphadenopathy was seen.

The patient was initially managed in the SARI ward as a case of SARI with a hyperglycemic emergency. Bronchoalveolar lavage was inconclusive. The patient was treated as severe COVID-19 in accordance with the protocol of our hospital and was given hydroxychloroquine $(400 \mathrm{mg} \mathrm{BD}$ on day 1 followed by $400 \mathrm{mg}$ for 4 days), dexamethasone (4 mg i.v. $\mathrm{BD})$, injection enoxaparin ( $0.4 \mathrm{ml} \mathrm{s} / \mathrm{c} \mathrm{OD})$, azithromycin (500 mg OD for 5 days), ceftriaxone (1 g i.v. BD), insulin (titrated as per random blood sugar level), and Vitamin C (500 mg OD).

The patient did not improve and was administered intravenous meropenem. There was a progressive fall in hemoglobin $(\mathrm{Hb})$ from $13 \mathrm{mg} / \mathrm{dL}$ to $8 \mathrm{mg} / \mathrm{dL}$. In view of falling $\mathrm{Hb}$, injection enoxaparin was stopped. Blood transfusions were done. The patient deteriorated rapidly and expired on the $14^{\text {th }}$ day of hospital admission.

\section{DISCUSSION}

The spikes present on the SARS-CoV-2 contain receptor binding domain that recognizes angiotensin-converting enzyme 2 (ACE-2) receptors present on epithelial surfaces of the lungs, heart, kidney, and intestines, which helps in an invasion into the host cell followed by biosynthesis, maturation, and release of new virus particles [3].
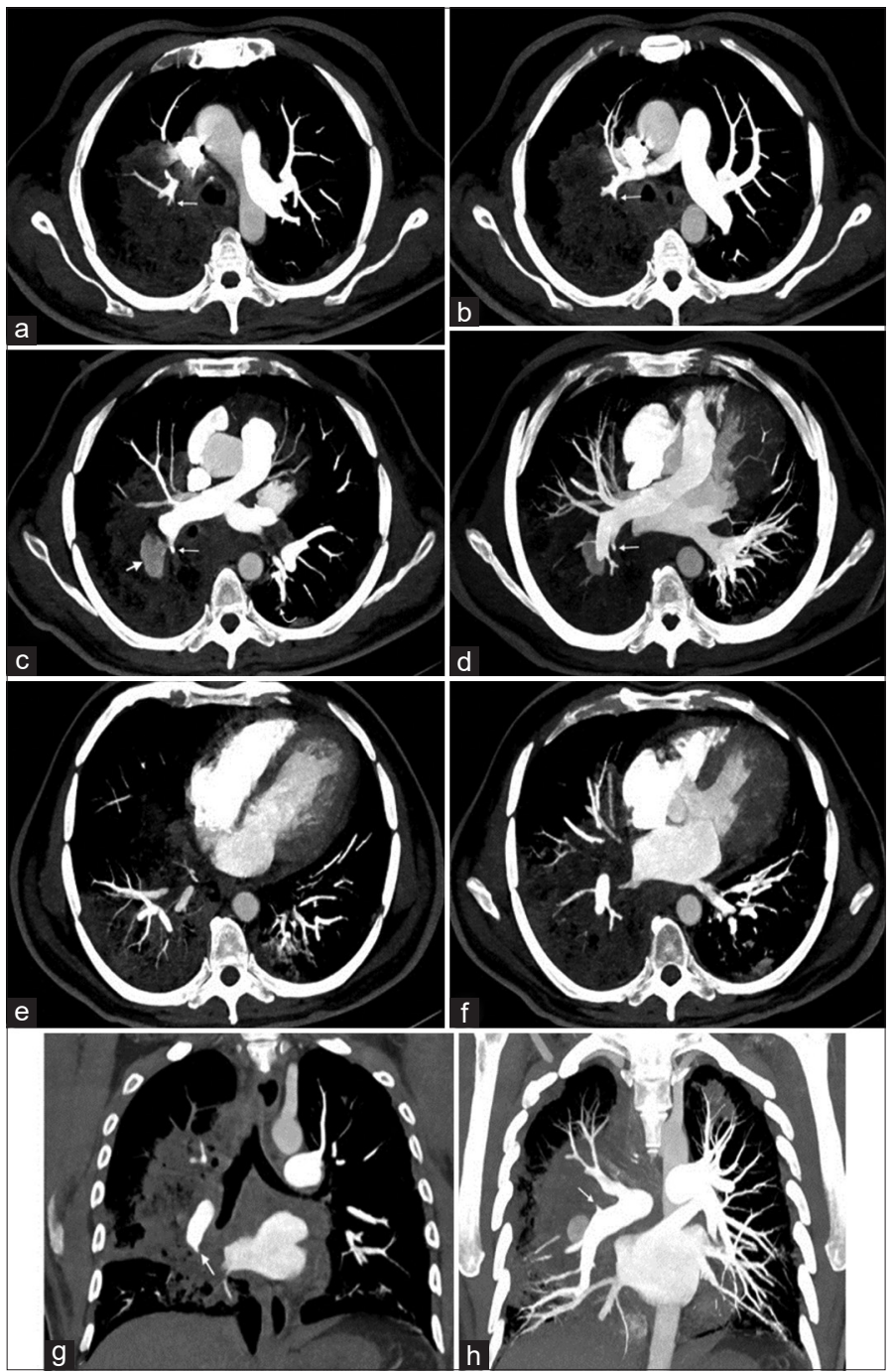

Figure 2: (a-f) CTPA MIP axial images. (a and b) Thrombosis involving segmental branch in the right upper lobe (arrows). Pulmonary trunk and bilateral main pulmonary arteries are normal. (c and d) Thrombus in segmental branch (long arrow) in the right lower lobe along with an aneurysm (short arrow) arising from the same segmental branch just proximal to the level of thrombus. A defect is seen in the anterior aspect of the aneurysm. Another thrombus involving segmental branch is seen on left side (curved arrow). (e and f) Normal right- and left-sided chambers with no evidence of the right ventricular strain. CTPA Coronal MPR (g) and MIP (h) images: showing other segmental thrombi on the right side (arrows) and paucity of vessels in the right upper and middle lobes as compared to the left side. Mild bilateral pleural effusion is also seen.

The pathogenesis of highly pathogenic human coronavirus has two basic components, that is, cytokine storm and microthrombosis [4]. A large number of inflammatory cells infiltrate the lungs of patients with severe COVID-19 disease leading to ARDS [3,5]. Endothelial inflammation results in a cascade of inflammatory response with complement activation, thrombin generation, recruitment of platelets and leukocytes along with initiation of innate, and adaptive immune responses that culminate in immunothrombosis, ultimately causing (micro)thrombotic complications such as DVT, pulmonary thrombosis, and stroke [6].

The majority of patients with COVID-19 have a mildto-moderate disease with a favorable prognosis and present 


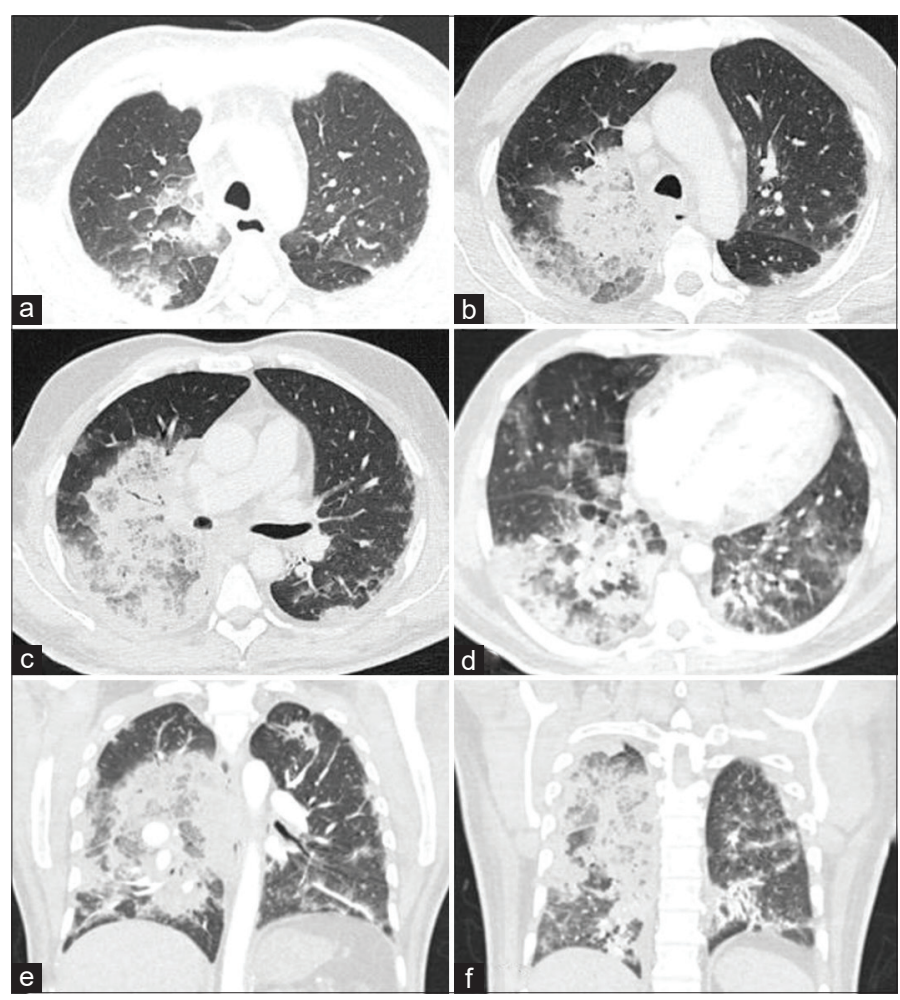

Figure 3: HRCT Lung window axial images (a-d) and coronal images (e and f) showing large confluent areas of consolidation and groundglass opacities with underlying interlobular thickening giving a crazy pavement appearance in parahilar location (with average Hounsfield Units 40) in the right upper, middle, and lower lobes that could be suggestive of pulmonary hemorrhage due to leaking aneurysm. Multiple scattered areas of consolidation and groundglass opacities are also noted in the both lung fields predominantly in peripheral areas.

primarily with fever, myalgia, fatigue, and dry cough. However, elderly patients and those with chronic underlying conditions and immunocompromised status may have worse outcomes and develop severe COVID-19 disease with the development of SARI leading to dyspnea and hypoxemia within 1 week after onset of the disease, which may quickly progress to ARDS or end-organ failure resulting in death $[1,2]$.

Chest CT in COVID-19 patients shows a pattern of multilobar ground-glass opacities or consolidation or both often with a bilateral and peripheral lung distribution. These findings are also the typical response in acute lung injury whereby an initial acute insult causes the development of ground-glass opacities that may coalesce to form consolidative lesions along with thickening of interstitium giving rise to radiological "crazy" paving sign. There is a predilection for the lung periphery. Evidence of dilated vessels has also been reported in several studies. CT appearance of COVID19 pneumonia may mimic pulmonary edema, hemorrhage, neoplasms, organizing pneumonia, pulmonary alveolar proteinosis, sarcoidosis, pulmonary infarction, interstitial lung diseases, and aspiration pneumonia. Bronchiectasis, pleural thickening, and subpleural involvement may be seen in the later stages of the disease. CT findings such as lymphadenopathy, pleural effusions, pulmonary nodules, and lung cavitation are usually absent [7].
Pulmonary thrombosis occurs as a complication of severe COVID-19 disease. Most often, it affects segmental and subsegmental vessels in segments showing consolidation and often involves both lungs and more commonly the right lung. According to a recent study by Covagna et al., severe COVID-19 pneumonia, as scored by $\mathrm{CT}$ and raised laboratory biomarkers (d-dimer, LDH, and CRP), was more commonly associated with pulmonary thrombosis. They also hypothesized that pulmonary thrombosis in COVID-19 is due to a hypercoagulable state due to the severe underlying lung inflammation caused by SARS- CoV-2 rather than common risk factors for PTE or DVT. Their study also revealed a higher incidence (40\%) of PTE in COVID-19 vis-à-vis $23-30 \%$ reported by earlier recent studies [8].

The WHO recommends the use of chest imaging to diagnose COVID-19 in symptomatic patients when RT-PCR testing is not available or has delayed results or when the initial RT-PCR test is negative but there is high clinical suspicion of COVID-19 [9]. An important issue associated with the real-time RT-PCR test is the risk of eliciting false-negative and false-positive results. It has been seen that many "suspected" cases with typical clinical symptoms of COVID-19 and characteristic CT images showed negative results on RT-PCR. False-negative results may occur because of many reasons like mutations in the primer and probe target regions in the SARS-CoV-2 genome, viral load kinetics in different anatomic sites of the patients, and faulty sampling procedures [10]. Therefore, a negative result does not entirely rule out the possibility of COVID-19 infection and should not be used as the only criterion for treatment or management decisions.

\section{CONCLUSION}

This case highlights the importance of CT pulmonary angiography with HRCT chest in cases where the RT-PCR test is delayed or negative despite strong clinical suspicion. In our case, typical findings of COVID-19 pneumonia were seen along with pulmonary thrombosis involving segmental vessels which confirms the clinical suspicion of COVID-19. However, the conundrum in our case was due to an incidentally noted pulmonary artery aneurysm. The question whether the aneurysm was due to COVID-19 or an incidental finding remains unanswered and needs to be studied.

\section{REFERENCES}

1. Huang C, Wang Y, Li X, Ren L, Zhao J, Hu Yi, et al. Clinical features of patients infected with 2019 novel coronavirus in Wuhan, China. Lancet 2020;395:497-506.

2. Chen N, Zhou M, Dong X, Qu J, Gong F, Han Y, et al. Epidemiological and clinical characteristics of 99 cases of 2019 novel coronavirus pneumonia in Wuhan, China: A descriptive study. Lancet 2020;395:507-13.

3. Yuki K, Fujiogi M, Koutsogiannaki S. COVID-19 pathophysiology: A review. Clin Immunol 2020;215:108427.

4. Channappanavar R, Perlman S. Pathogenic human coronavirus infections: Causes and consequences of cytokine storm and immunopathology. Semin Immunopathol 2017;39:529-39.

5. $\mathrm{Xu} \mathrm{Z,} \mathrm{Shi} \mathrm{L,} \mathrm{Wang} \mathrm{Y,} \mathrm{Zhang} \mathrm{J,} \mathrm{Huang} \mathrm{L,} \mathrm{Zhang} \mathrm{C,} \mathrm{et} \mathrm{al.} \mathrm{Pathological}$ findings of COVID-19 associated with acute respiratory distress syndrome. Lancet Respir Med 2020;8:420-2.

6. McFadyen JD, Stevens H, Peter K. The emerging threat of(Micro) thrombosis 
in COVID-19 and its therapeutic implications. Circ Res 2020;127:1-6.

7. Hosseiny M, Kooraki S, Gholamrezanezhad A, Reddy S, Myers L. Radiology perspective of coronavirus disease 2019 (COVID-19): Lessons from severe acute respiratory syndrome and middle east respiratory syndrome. Am J Roentgenol 2020;214:1078-82.

8. Cavagna E, Muratore F, Ferrari F. Pulmonary thromboembolism in COVID19: Venous thromboembolism or arterial thrombosis? Radiology 2020;2:1-4.

9. World Health Organization. Use of Chest Imaging in COVID-19: A Rapid Advice Guide, WHO Reference Number. Geneva: World Health Organization; 2021. Available from: WHO/2019-nCoV/Clinical/Radiology_ imaging/2020.1. [Last accessed on 2021 Mar 20].
10. Tahamtan A, Ardebili A. Real-time RT-PCR in COVID-19 detection: Issues affecting the results. Expert Rev Mol Diagn 2020;20:453-4.

Funding: None; Conflicts of Interest: None Stated.

How to cite this article: Agarwal A, Kumar N. CT chest with pulmonary angiography as a diagnostic tool in clinically suspected RT-PCR-negative COVID-19 pneumonia with pulmonary artery aneurysm. Indian J Case Reports. 2021;7(3):79-82. 